\title{
Defined Repeat Activity Until Rule
}

National Cancer Institute

\section{Source}

National Cancer Institute. Defined Repeat Activity Until Rule. NCI Thesaurus. Code C93474.

A relationship between a defined repeating activity and one of the following that may trigger the repeating activity to stop, where all items are part of a global library: Another defined activity where the repeating activity stops if this other activity occurs; A defined observation result where the repeating activity stops if this observation result occurs; $\mathrm{A}$ defined criteria group where the repeating activity stops if this group logically evaluates to true based on its components. 